\title{
Ubrogepant: a new era in migraine treatment
}

\author{
Sangeeta Bhanwra ${ }^{1 *}$, Kaza Ahluwalia ${ }^{2}$
}

\author{
${ }^{1}$ Department of Pharmacology, Government Medical College and Hospital, Chandigarh, Punjab, India \\ ${ }^{2}$ Senior Drug Safety Physician, Parexel International, Chandigarh, Punjab, India
}

Received: 11 February 2020

Revised: 13 March 2020

Accepted: 18 March 2020

\section{*Correspondence:}

Dr. Sangeeta Bhanwra,

Email: doc_sangeeta@yahoo.com

Copyright: (C) the author(s), publisher and licensee Medip Academy. This is an open-access article distributed under the terms of the Creative Commons Attribution Non-Commercial License, which permits unrestricted non-commercial use, distribution, and reproduction in any medium, provided the original work is properly cited.

\begin{abstract}
Migraine is the most common neurological disorder that leads to incapacitating neurological symptoms. Acute attacks of migraine have been dealt with effectively with non-steroidal anti-inflammatory drugs (NSAIDs), ergot derivatives and triptans since long, but their use is limited by various adverse effects. Recent studies have shown that a neuropeptide, calcitonin gene related peptide (CGRP) plays a major role in pathophysiology of migraine by increasing the pain perception, both at the peripheral and central nervous system levels. So, in the last few years, some CGRP antagonists have found their way in the treatment of both episodic and chronic migraine. Ubrogepant is the first oral CGRP antagonist, that was approved by USFDA (United States food and drug administration) in December 2019, for the acute treatment of migraine with or without aura. It is more potent than the earlier CGRP receptor antagonists, has a good oral bioavailability and the risk of hepatotoxicity is also lesser than the previous Gepants.
\end{abstract}

Keywords: Ubrogepant, CGRP antagonist, Migraine

\section{INTRODUCTION}

Migraine is the most common neurological disorder with incapacitating neurological symptoms causing ability worldwide. ${ }^{1}$ It commonly affects adults (age 18-44 years), more so females. ${ }^{2}$ It is characterized by episodic headaches, with photo and phono-phobias and autonomic symptoms. It might be preceded by an aura. ${ }^{3}$ The recent insights into the pathophysiology of migraine have led to unraveling of neurophysiological mechanisms and neurotransmitters paving way for newer clinical approaches for migraine patients. The treatment of migraine includes both acute and preventive therapies. Acute attacks of migraine have been since long dealt effectively with non-steroidal anti-inflammatory drugs (NSAIDs), ergot derivatives and triptans, with their use limited by adverse effects. ${ }^{4}$ The patients with chronic migraine have been advocated preventive therapy with drugs like beta blockers, antidepressants and calcium channel blockers. Since these treatments are fraught with serious adverse effects and limited effectiveness, new frontiers for managing migraine have been investigated. It is now evident that migraine pathophysiology cannot be pinpointed to either vascular or neuronal theory, and that neuropeptides like calcitonin gene related peptide (CGRP) increase the pain perception in migraine, both at periphery and central nervous system levels. With this recently established role of CGRP in migraine, CGRP antagonists found their way in the treatment of both episodic and chronic migraine. ${ }^{5}$

There are two different classes of drugs that help in management of migraine by blocking CGRP: CGRP antagonists and monoclonal antibodies which target either CGRP or CGRP receptors. The CGRP antagonists are alternatively called "Gepants". The first drug in this group to be investigated was olcegepant followed by telcagepant. Among this group, ubrogepant is the first 
oral CGRP antagonist, approved in December 2019, for the acute treatment of migraine, with or without aura, by USFDA (United States food and drug administration). ${ }^{6}$

\section{ROLE OF CGRP IN MIGRAINE}

Recent studies have shown that CGRP neuropeptide plays a major role in pathophysiology of migraine. It is released from activated trigeminal sensory nerves, along with causing dilatation of both intracranial and extracranial blood vessels. ${ }^{7}$ Additionally, CGRP modulates vascular nociception centrally. Three lines of clinical evidence support the role of CGRP in migraine. It was first seen in a study that CGRP levels were increased in the jugular outflow during migraine attacks, later confirmed in induced migraine experiments. ${ }^{8}$ It was further seen that intravenous injection of CGRP led to experimentally induced migraine, thereby confirming the role of CGRP in its pathophysiology. ${ }^{9}$ Finally, it was seen that CGRP receptor antagonists were effective in decreasing both pain and other symptoms of migraine, consolidating its role in pathophysiology of migraine. ${ }^{8}$

\section{UBROGEPANT: AN ORAL CGRP ANTAGONIST}

It is an oral CGRP antagonist, approved by USFDA (December 2019) for the treatment of acute migraine, with or without aura. It is a competitive antagonist with high affinity, potency, and selectivity for the human CGRP receptor. It is more potent than the earlier CGRP receptor antagonists, with added benefit of lesser dose for similar therapeutic effect and decreased associated risk of hepatotoxicity unlike the previous gepants. It is not recommended for the prevention of migraine. ${ }^{10}$ The basic mechanism of action of ubrogepant is potent blocking of human $\alpha$-CGRP-stimulated cAMP (cyclic adenosine monophosphate) responses in human CGRP receptor. ${ }^{11}$

The dose recommended is $50 \mathrm{mg}$ or $100 \mathrm{mg}$, to be taken orally, as needed, with or without food. If needed, the dose can be repeated in two hours after the first dose. The maximum recommended daily dose should not exceed $200 \mathrm{mg} \cdot{ }^{12}$

After administration, it is rapidly absorbed with peak plasma concentration in 1.5 hours and has $87 \%$ plasma protein binding. The mean apparent volume of distribution of ubrogepant is $350 \mathrm{~L}$. The major metabolism takes place through CYP3A4 enzyme, thereby making it prone to drug- drug interactions. Hence, ubrogepant should not be used concomitantly with CYP3A4 inhibitors or inducers as it might affect its efficacy. Also, since ubrogepant is a substrate of BCRP (breast cancer resistance protein) and $\mathrm{P}$-gp ( $\mathrm{P}$ glycoprotein) efflux transporters, hence use of this drug with inhibitors of these transporters is also cautioned against. The elimination half-life is 5-7 hours, with excretion primarily through biliary/ fecal route and very less through renal route. Studies have shown that dose adjustment is not required in the patients with mild or moderate renal impairment. However, it was seen that ubrogepant exposure levels increased by mild/ moderate and severe hepatic impairments, requiring dose adjustments. ${ }^{12}$

Regarding use in pregnancy, only data from animal studies have been populated, which showed adverse embryofetal and developmental effects, if given during pregnancy or lactation. The safety of use has not been established in pediatric population as of now. ${ }^{12}$

\section{EFFICACY AND SAFETY DATA}

The effectiveness of ubrogepant in the treatment of acute migraine has been demonstrated in two clinical trials. In ACHIEVE 1 and ACHIEVE II trials, patients on ubrogepant achieved significant relief of most bothersome symptoms along with headache pain, within two hours of administration, compared to placebo. ${ }^{13,14}$

In an open label extension study, safety of ubrogepant was assessed and the most common adverse reactions reported were nausea, somnolence and dry mouth and these were mostly seen with $100 \mathrm{mg}$ dose. ${ }^{12,15}$ Other serious adverse effects reported in ubrogepant group within 30 days of administration were appendicitis, spontaneous abortion, pericardial effusion, and seizure. ${ }^{13}$

\section{CONCLUSION}

Ubrogepant is a highly efficacious and safe anti-migraine medication as established by the safety and efficacy studies. Unlike earlier gepants, it is not hepatotoxic and has a good oral bioavailability. Further clinical trials are needed to shed more light on efficacy and safety profile of this drug, in comparison to other anti-migraine medications.

\section{Funding: No funding sources \\ Conflict of interest: None declared \\ Ethical approval: Not required}

\section{REFERENCES}

1. Global Burden of Disease Study 2013 Collaborators. Global, regional, and national incidence, prevalence, and years lived with disability for 301 acute and chronic diseases and injuries in 188 countries, 19902013: a systematic analysis for the Global Burden of Disease Study 2013. Lancet. 2015;386(9995):743800.

2. Saper J. Migraine research foundation. Available at: https://migraineresearchfoundation.org/aboutmigraine/migraine-in-women/. Accessed on 07 February 2020.

3. Edvinsson L. Role of CGRP in Migraine. Handb Exp Pharmacol. 2019;255:121-30.

4. Humphrey PP, Feniuk W, Perren MJ, Beresford IJ, Skingle M, Whalley ET, et al. Acad Sci. 1990;600:587-8. 
5. Russo AF. Calcitonin gene-related peptide (CGRP): a new target for migraine. Annu Rev Pharmacol Toxicol. 2015;55:533-52.

6. Kahn J. FDA approves new treatment for adults with migraine. 2019 Available at: https://www.fda.gov/ news-events/press-announcements/fda-approvesnew-treatment-adults-migraine. Accessed on 08 February 2020.

7. Villalon CM, Olesen J. The role of CGRP in the pathophysiology of migraine and efficacy of CGRP receptor antagonists as acute antimigraine drugs. Pharmacol Ther. 2009;124(3):309-23.

8. Ho TW, Edvinsson L, and Goadsby PJ. CGRP and its receptors provide new insights into migraine pathophysiology. Nat Rev Neurol. 2010;6(10):57382.

9. Lassen LH, Haderslev PA, Jacobsen VB, Iversen HK, Sperling B, Olesen J. CGRP may play a causative role in migraine. Cephalalgia. 2002;22(1):54-61.

10. Scott LJ. Ubrogepant: First Approval [published online ahead of print, 2020 Feb 5]. Drugs. 2020;10:1007.

11. Moore E, Burgey CS, Fraley M. Characterization of ubrogepant: A potent and selective antagonist of the human calcitonin gene-related peptide receptor [abstract]. Neurology. 2019;92:10-21.
12. Allergan. Ubrogepant: Label-FDA [Internet]. 2019. Available at: https://www.accessdata.fda.gov/ drugsatfda_docs/label/2019/211765s000lbl. Accessed on 09 February 2020.

13. Dodick DW, Lipton RB, Ailani J. Ubrogepant for the Treatment of Migraine. N Engl J Med. 2019;381(23):2230-41.

14. Lipton RB, Dodick DW, Ailani J, Lu K, Finnegan M, Szegedi A, et al. Effect of Ubrogepant vs Placebo on Pain and the Most Bothersome Associated Symptom in the Acute Treatment of Migraine: The ACHIEVE II Randomized Clinical Trial. JAMA. 2019;322(19):1887-98.

15. Allergan. Safety, Tolerability and Drug Interaction Study of Ubrogepant with Erenumab or Galcanezumab in Participants with Migraine [Internet]. Available at: https://clinicaltrials.gov/ct2/ show/NCT04179474. Accessed on 09 February 2020.

Cite this article as: Bhanwra S, Ahluwalia K Ubrogepant: a new era in migraine treatment. Int J Basic Clin Pharmacol 2020;9:819-21. 